Secondary syphilis gives rise to secondary skin affections of the conjunctiva, which attend it on other parts of the body. (Swanzy, "Diseases of the Eye," 1892).

In cases where the face is the seat of papular or tubercular eruptions, a corresponding condition may at times, though rarely, be observed upon the lining membrane of the lids (De Wecker). The papular syphilide appears from the third to the sixth month. Unless they ulcerate, the papules leave no scars. They frequently leave pigmented areas behind (Keyes).

The following notes of my case are incomplete, as the patient unexpectedly discontinued his attendance:

$\mathrm{N}$. T., age 24 , male, colored, came to my clinic at the Charity Hospital in November, 1894, on account of an inflammation of the left eye, which had somewhat annoyed him for a week, with a feeling as if something had got into his eye. At the limbus of the cornea were four nodules implanted in the conjunctiva; one on the nasal side, one on the temporal, and two below and within 3 m.m. of each other. The lateral nodules were 4 m.m. in diameter by $2 \mathrm{~m} . \mathrm{m}$. in height. The lower $3 \mathrm{~m} . \mathrm{m}$. in diameter by $2 \mathrm{~m} . \mathrm{m}$. in height. All circular, with a finely granular apex, and of the red color of granulation tissue. The papillary reaction, the iris and the cornea were normal. $\mathrm{V}=20-50$. The refraction was not tested. In the vicinity of the nodules there was moderate hyperemia of the conjunctiva. Very little p.in had been complained of. Two very small ulcers $(1 \mathrm{~m} . \mathrm{m}$. in diameter) were situated on the outer third of the anterior edge of the upper lid. The pre-auricular glands were not enlarged. A history was given of the appearance on the face, at the age of 13, of an eruption which disappeared and left no pitting and relapsed once in about three years. During July last he contracted a chancre, which was followed by swelling of the inguinal glands, and a secondary papular eruption on the skin. The cheeks and forehead presented a thickly covered, mixed acne-like and papular eruption, arranged here and there in somewhat irregularly concentric groups. Numerous depressed hyper-pigmented scars were scattered among the papules. The covered parts of the body were not examined.

During the second week of treatment the papules of conjunctiva diminished one-half in size, and around the two lower edges of the conjunctiva a pigmented zone was first seen.

The diagnosis of a secondary papular syphilide of the conjunctiva was based upon the syphilitic history of the case, the associated papular syphilide of the face, the exclusion of other lesions of the conjunctiva, and the rapid resolution of the nodules without ulceration under the mixed treatment and the application to the eye of an ointment of hydrarg. ox. rub. grs. ii, vaselin $3 j$ rubbed in for five minutes daily, by massage of the lids.

The patient was referred to Dr. Baum, Professor of Dermatology, Post-Graduate Medical School, who confirmed the diagnosis and prescribed the constitutional treatment. The rapid progress toward recovery (in two weeks) probably accounts for the early vanishing of the possessor of our rare specimen.

$$
\text { DISCUSSION. }
$$

Dr. C. D. Wescotr-As Dr. Coleman has said, these cases are very rare; it has never been my fortune to see one such as the Doctor has described. The only syphilitic lesions of the conjunctiva I have seen are the simple chancre, of which I have seen one case, and one case of gumma. It does not seem possible that there can be an error of diagnosis in this case. I do not know of anything that affects the conjunctiva which would give a similar appearance, even without the confirmative syphilitic history and other lesions. I congratulate the Doctor on having had an opportunity to observe such a case.

DR. F. W. Coldeman-I would say with regard to chancre that they are not so very infrequent upon the edge of the lid, and chancres upon the lids might be easily mistaken for epitheliomata. I have had the misfortune to remove from the lid what I thought was an epithelioma and had a chancre turn up very near it within ten days, which was cured with iodid of potassium. This ease, by the way, was diagnosed by the syphilologists as an epithelioma. Gumma is not so rare although it seldom manifests itself in the conjunctiva, but not so rarely in the iris and ciliary body involving the sclera and therefore appearing externally as a nodule. That the Doctor does not refer to. The gumma that limits itself and is situated primarily in the conjunctiva is an exceosively rare disease. Up to 1878 Bull makes a resume of all the cases he has had access to and describes them fully, but does not describe a single case of papule. The "Indicus Medicus" from 1879 to date only records a single case by title of papule of the conjunctiva.

DR. C. W. HATLEY-Speaking of gumma of the conjunctiva reminds me of a case I saw in a bospital in Europe, which was diagnosed gumma of the conjunctiva and was treated with mercurials very faithfully for a long time, but it did not seem to improve and finally the surgeon concluded to operate upon it. When he made his incision through what he at first supposed was a gumma he removed three pieces of putty. It was impossible to get any history of how the putty came there, the patient said she did not know; either she did not want to tell or did not know how it got there.

\section{A CASE OF TACHYCARDIA COMPLICATING CHLOROFORM ANESTHESIA.}

Read before the Louisville Academy of Medicine, March 18, 1895. BY HENRY E. TULEY, A.B., M.D.

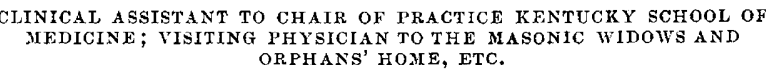

The following is the report of a case of tachycardia, complicating chloroform anesthesia. The man was a patient of Dr. Mathews, and was to be operated upon for fistula. He was about 30 years of age; a strong, vigorous man, and a good subject for chloroform anesthesia. The following previous history was detailed after his regaining consciousness. He had had repeatedly, attacks of heart trouble, a greatly accelerated heart's action being the chief symptom, with once or twice loss of consciousness during the attack. He is a slave to smoking, using the strongest tobacco, uses liquor sometimes to excess and drinks strong coffee. Face was florid, but heart's action normal, pulse 84 , with no organic lesion. He was very nervous when he got on the table, and asked that his heart be examined but said nothing of his previous history. The anesthesia was begun at 3:06 P.M., with chloroform, on the Esmarch inhaler, drop by drop method. The exciting stage was quite severe, the chloroform was pushed and in three minutes he was relaxed, it having taken 25 -10 drachms to bring him to the surgical degree, taking fourteen minutes; in all 5 drachms were used.

The operation was begun at $3: 20$ P.M; the pulse and respiration were normal. At about $3: 42$, after the operation was nearly completed, the chloroform mask was removed, as the pupils were well contracted and the patient fully anesthetized. At $3: 44$ without any warning whatever, the pulse from an even regular one of about 80 or 90 suddenly began beating too fast to count, more than 200 . The respirations remained unimpaired. We administered 1-100 grain of nitroglycerin hypodermatically, the dressing was hastily applied and the patient put to bed. While his pulse was in this condition and before he was removed from the table he raised his head and said: "Well, I'm glad I'm through with that chloroform." During the next hour 1-25 grain of nitroglycerin was administered, 1-100 grain of atropia and 1-32 of strychnia, without any effect as to lessening the rapidity of the heart beats. Hot water bags were applied to feet, and over the heart. Only at one time, and then for only four or five minutes, did he complain of dyspnea. He was convinced of the fact that he was going to die. The radial pulse all of this time, one hour, felt as if there were a constant current of blood under your finger, no pulsation was distinguishable, but the ventricular contraction was easily made out, much too rapid to count. His respirations were unimpaired, breathing through his nose, skin relaxed and moist, mouth dry from the atropia and finger nails pink. He was seen at $4: 20$ by Dr. Marvin in consultation; 1/4 grain of morphia was given at his suggestion hypodermatically, ice was substituted over the precordia, and in twenty minutes the pulsations could be felt, not counted at the wrist, but the ventricular contractions could be counted 160 to the minute. His pulse remained in this condition until 7 P.M., when it 
again became rapid, beating 200 to the minute. A second dose of morphia, $1 / 4$ grain was given hypodermatically, and at 8 P.M., his pulse was 97 , and the patient sleeping quietly. having vomited some water which had been taken in the shape of crushed ice. During the night he slept at intervals and the hourly pulse was as follows, beginning at 7 P.M.: Two hundred, $97,98,92,88,80,80,81,78,78$. At midnight one teaspoonful of aromatic spirits of ammonia was given.

The case seems to be a unique one as well as in. structive. Here was a man who apparently was a good subject for anesthization and without warning the above complications arose. It teaches us that the rule laid down by most authors as to the careful watching of the respiration alone is erroneous. Had this man's condition not been noted, and the operation only partially completed, and had more chloroform been given, I believe he would have died. Hence the practical conclusion, watch the heart always, as well as the respiration. The second lesson is the danger of being too zealous in the application of restoratives, owing to anxiety as to the safety of your patient. There was in all probability too much given this man.

This case is entirely unique in the writer's experience, nor have $I$ seen any such case in the literature of the subject.

\section{DISCUSSION.}

Dr. BAILEY-The subject of anesthesia is a big one. As to the case reported, I would criticise it first, because Dr Tuley said the patient was a vigorous healthy man; I find these the worst cases. I think his condition was peculiar and not developed by the anesthesia in the light of his present condition.

There was a bad nerve force present. As to the management of the case, with the rapid heart, nitroglycerin should not have been given. I should rather have chosen digitalis or digitalin because of its influence on the inhibition. I should have used moderate doses of morphin-should not have ventured 1-4 grain but have given 1-8 grain and waited for effects.

I think constantly of the possible evils of the Hyderabad Commission, in recommending that the respiration be watched and not the pulse. Notwithstanding that report, I am more anxious to watch the pulse, and I endeavor to lojk after both. I disregard the pulse in etherization, but not in chloroform. I am sure $\mathrm{I}$ have given chloroform in more than one thousand cases, and I am firmly convinced of that fact.

Dr. F. C. WILSON-The case reported is an interesting one and shows the necessity of careful watching. To watch the pulse and respiration both, the anesthetist has his hand full. He should also watch the countenance. Anesthesia must be profound in operations upon the rectum, and be cause of that fact the danger is enhanced. If the previous attacks had been known in this patient, in all probability the case would have been watched for a recurrence, but doubt if the case could have been watched more closely, or the tachycardia noticed earlier than was done by Dr. Tuley. Like Dr. Bailey I should not have given nitro-glycerin, but think that digitalin was indicated; strychnia also, as was given, morphia and perhaps spartein; the latter given previous to the administration of the anesthetic fortifies the beart, and those cases in which I have used it have done well, the heart maintaining its strength throughout the anesthesia.

Dr. BAILEY-I would like to state that as to complete anesthesia, it is difficult to bring anesthesia to a proper stage for divulsion of the sphincter, and there apparently is pain. Lauder Brunton said that no operation should be done unless there was complete anesthesia. It may be possible that the case was not fully anesthetized, and the reflex caused the tachycardia. No operation should be done unless the anesthesia is complete.

$D_{R}$. VANCE-I believe that in local anesthesia the shock is not so profound. We used to see great shock from the removal of a toe nail under general anesthesia. Since cocain has been introduced we see less of it. the nerves are benumbed locally, and they are prevented from transmitting any shock. I believe that all operations should be done under complete anesthesia. The greatest accidents are when we operate under primary anesthesia. Local anesthesia proves that the shock is reflex, as it is in the divulsion of the rectum.

Dr. W. C. ChapMan-It has been noticed how frequently tachycardia occurs in tobacco users. I never examine tobacco users that I don't find them with a pulse rate of ten to twenty above normal. Tachyeardia is not present at all times, and may be brought on by other causes, as a latent malaria may develop after a surgical operation, so an indigestion and excesses may cause tach ycardia.

Dr. DeGAN-I have had two fatal cases under chloroform anesthesia. Neither used tobacco, for they were both ladies both occurred within a week of each other. The first one was an operation for ovariotomy ; there was only one inbalation, the patient called for "a candle, a candle," being a Roman Catholic, in one breath. The lips became livid, the heart's action stopped. I thought that perhaps it was partially from pressure by the large tumor, and plunged my knife through the abdominal wall and relieved that by letting cut the fluid, but the patient never rallied. This case was on Sunday, and the following Tuesday I wished to divulse a rectum for fissure. The patient was not excited, her heart was good. pulse regular. She was rather inclined to be fat, but took the anesthetic like a baby. When Dr Guest said she was ready, I introduced my thumbs and divulsed; there was no pain, no resistance, chloroform was withdrawn, and the patient was sponged off. In half a minute, Dr. Guest seemed concerned, and said the patient was not breathing well. I noticed some lividity of the face. She was suspended, artificial respiration performed, and at no time during the forty-five minutes we worked with her did she show pulse or respiration. She was healthy, a nontobacco user, and drank little tea or coffee. We can not tell when these cases will do badly. The shock could not have been due to the divulsion, and Dr. Guest estimated that only 30 minims had been used altogether.

DR. J B. BulLITT-Dr. Wright, of Bowling Green, has in the last few months reported several cases of the new.born asphyxiated, in whom respiratory effort was established by the introduction of the tip of the index finger in the anal orifice, producing thereby a dilatation of the sphincter. There was complete asphyxiation, absolutely no respiratory effort, but immediately on dilatation of the sphincter ani, respiration was established and the infant began to cry lustily. He also suggested the adoption of similar means to restore respiratory effort in unfortunate cases of anesthetization. This fact would appear to throw some light on the subject in hand. It is generally conceded that death from chloroform occurs primarily from respiratory failure, the heart failure being secondary. It hardly seems probable that dilatation of the sphincter ani could produce respiratory effort on the one hand (asphyxia neonatorum) and yet produce a paralysis of effort on the other (chloroform narcosis). There seems to be here an incompatibility of effects. It would seem probable, that what really happens is this: divulsion of the sphineter before narcosis is surgically complete causes deeper respiratory effort, thereby a largely increased amount of the anesthetic is thrown suddenly into the circulation, so that the centers are overwhelmed and respiration suddenly ceases. It is not the "shock" per se, that has done the mischief, but its respiratory effects. In such a case, if the anesthetic were withdrawn and the divulsion of the sphincter ani again practiced, it would seem quite probable that respiratory effort would again be stimulated as suggested by Dr. Wright.

Dr. Roman-We never know when accidents will occur. If deaths are more likely occur from partial anesthesia, why do they not occur without any anesthesia in minor operations? Certainly if a little pain is so dangerous, we would have many deaths where no anesthetic is given. I do not believe Dr. Bailey's explanation the true one. I think it is best to have light anesthesia in the condition of shock. I am satisfied that the danger is in proportion to the amount of chloroform vapor in the blood at any given time. There have been more deaths reported in the last few years than ever before in the history of chloroform. In Germany, chloroform used to be given almost exclusively, but ether is now being extensively used. There have been five or six deaths in Louisville in the past eighteen months from chloroform, and there were none for five years previous that $I$ remember. It may be that the chloroform we use now is not so good, but the chemists say it is better. A statement was made recently by the Professor of Therapeutics in the Johns Hopkins Hospital, that if we give chloroform to a dog once it bears it well, and will have no bad effects, but if it is given the second time it kills, but this is not so of ether. 
Hare, in a paper on chloroform, makes the statement that instead of lowering the head it should be thrown forward as that position opens the epiglottis. Notwithstanding chloroform has been a favorite in Louisville for so many years. I am an advocate of ether. It can be incontestably shown that ether is safer than chloroform.

DR. I. N. BLoom-The history of anesthesia shows ether to be the safer. It is rare that death occurs in complete anesthesia. Statistics will prove this.

DR. DvaAx--I would like to add that I saw a patient operated on by Dr. Weir for a nephrectomy, die under the anesthetic, having taken it twenty-one times before.

Dr. TULFY - I simply want to state that nitro-glycerin was given in the case reported, because there was a good effect noticed from it, in that the pulsations at the wrist could be felt after each dose. Digitalin was thought of but none was at hand. The divulsion was practiced without causing any impression upon the patient.

\section{THE NORMAL SITUATION OF THE GALL BLADDER IN MALES.}

BY JOHN B. HAMILTON, M.D. CHICAGO.

To find the gall bladder, draw a line from the anterior superior spinous process of the ilium to the center of the xiphoid appendix. Intersect this with a line from the umbilicus to the tenth costo-cartilaginous junc-

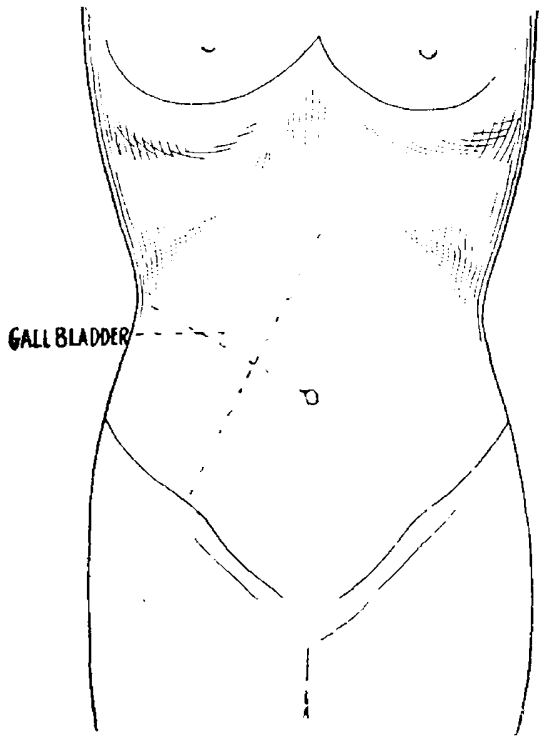

tion. In the right upper triangle near the apex, but nearer the right oblique line, the fundus of gall cyst will be found. A needle thrust through the abdomen at the point indicated, will usually transfix the gall bladder. The position of the gall bladder varies with the changes in the position of the liver; in enlargements it is pushed downward, and in some cases deflected to the right.

\section{ORIGINAL INVESTIGATIONS ON THE NATURAL HISTORY, (SYMPTOMS AND PATHOLOGY) OF YELLOW \\ FEVER. 1854-1894. \\ BY JOSEPH JONES, M.D., LL.D. NEW ORLEANS, LA. \\ (Continued from page 520.) Chapter VIII.}

EFFECTS OF JAUNDICE UPON THE FREQUENCY OF THE PULSE.

Case 1.-Malarial fever, dysentery and jaundice. A. F age 32 ; native of Germany. Entered Charity Hospital Dec.
29,1875 . Had had intermittent fever. Says that he has been sick with intermittent fever and dysentery for fourteen days. Upon entrance presented a pale sallow, bloodless hue, and suffered from painful discharges from the bowels, of mucus and blood at regular intervals. On January 1, the patient showed a distinct yellow tinge of the skin and conjunctiva of the eyes, which gradually increased to the most intense jaundice in the course of ten days. The urine was heavily loaded with bile, but free from albumin. The pulse which had been rapid and feeble, ranging from 100 to 120 , became much slower after the supervention of the jaundice. The effect of the bile in the blood upon the frequency of the pulse is shown in the following table:

\begin{tabular}{|c|c|c|c|c|c|c|c|}
\hline \multirow{2}{*}{$\begin{array}{c}\text { Date. } \\
1875\end{array}$} & \multicolumn{2}{|c|}{ Pulse. } & \multicolumn{3}{|c|}{ Resp. } & \multicolumn{2}{|c|}{ Temp. } \\
\hline & M. & E. & 1 & M. & E. & M. & E. \\
\hline $\begin{array}{r}\text { Jan. } 13 \\
14 \\
15 \\
16 \\
17 \\
18 \\
19 \\
20 \\
21 \\
22 \\
23 \\
24 \\
25 \\
26 \\
27 \\
28 \\
29 \\
90 \\
31 \\
\text { Feb. } 1 \\
2\end{array}$ & $\begin{array}{r}70 \\
100 \\
80 \\
70 \\
72 \\
80 \\
76 \\
76 \\
72 \\
70 \\
72 \\
72 \\
60 \\
72 \\
60 \\
68 \\
68 \\
68\end{array}$ & $\begin{array}{r}70 \\
70 \\
80 \\
80 \\
100 \\
92 \\
92 \\
80 \\
88 \\
84 \\
72 \\
68 \\
80 \\
72 \\
76 \\
72 \\
64 \\
64 \\
68 \\
68\end{array}$ & 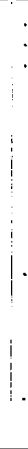 & $\begin{array}{l}28 \\
24 \\
32 \\
24 \\
28 \\
20 \\
24 \\
32 \\
28 \\
.9 \\
36 \\
40 \\
36 \\
32 \\
28 \\
40 \\
32\end{array}$ & $\begin{array}{l}32 \\
36 \\
32 \\
32 \\
32 \\
32 \\
36 \\
20 \\
24 \\
24 \\
28 \\
20 \\
40 \\
36 \\
32 \\
32 \\
36 \\
32 \\
32 \\
. \quad .\end{array}$ & \begin{tabular}{|c|c} 
&. \\
99 & 0 \\
101 \\
98 \\
99.5 \\
99.5 \\
98.5 \\
99.5 \\
99.5 \\
99.5 \\
97.5 \\
99.5 \\
98.5 \\
99 \\
99.5 \\
99.5 \\
98.5
\end{tabular} & $\begin{array}{c}99 \\
101 \\
100 \\
101 \\
104 \\
102 \\
103.5 \\
103.5 \\
103.5 \\
101 \\
100.5 \\
100 \\
99.5 \\
98.5 \\
99 \\
100 \\
99.5 \\
100 \\
99 \\
99.5 \\
. . .5\end{array}$ \\
\hline
\end{tabular}

The periodic variations of the temperature in this case were regarded as the result of the action of the malarial poison. It will be observed that in this case after the supervention of jaundice, the pulse was only 100 , with a temperature of 104 degrees $F$., and on January 20 , the pulse was only 80 , with a temperature of 103.5 degrees $F$. On the 30th the pulse was only 60 , notwithstanding the continuance of the dysentery, with frequent discharges from the bowels.

The treatment of this case consisted mainly in the rigid adherence to milk and rice diet and beef tea, and the employment at regular intervals of a mixture of quinin, subnitrate of Bismuth and Dover's powder; in the proportion of 3 grains each of quinin and Dover's powder, and 10 grains subnitrate of bismuth, every three, four or six hours. These powders were also alternated with a simple mixture of syrup of morphia and subnitrate of bismuth, or with a mixture composed of equal parts of the tinctures of catechu, opium, camphor, capsicum and Peruvian bark, a teaspoonful in a wineglass of water every three or six hours. Port wine and alcoholic stimulants in small quantities were also found to be beneficial. This was a protracted and tedious case, but the patient was finally restored to health and was discharged in the month of March.

Case 2.-Malignant scarlet fever attended with jaundice; slow pulse, recovery. Adam Grethiel, age 19; native of New Orleans. Entered Charity Hospital Feb. 11, 1876. February 11, deep scarlet eruption; where the eruption is not very great the skin presents a yellowish tinge. Conjunctiva of eyes yellow. Mucous membrane of throat and tonsils swollen and ulcerated. Tongue dry and red ; patient swallows with difficulty; hemorrhage from the mouth and gums; wild delirium; necessary to confine the patient to bed. Has been sick about one week. Abscesses are beginning to form near the elbows and at the extremities of the fingers ; P.M. pulse 100, respiration 48, temperature 103.5. February 12, A.M., pulse 100 , respiration 36 , temperature 102 ; P.M., pulse 108 , respiration 28 , temperature 103 . Desquamation is beginning, and as the scarlet color diminishes, the deep yellow color becomes more manifest. February 13, A.M., pulse 100, respiration 36 , temperature $1000^{\circ}$ P.M. pulse 124 , respiration 36, temperature 103.5. February 14, A.M., pulse 88, respira- 